\title{
LAS PÁGINAS WEB DE LOS SERVICIOS DE VIGILANCIA EPIDEMIOLÓGICA COMO INSTRUMENTO DE DIFUSIÓN DE LA INFORMACIÓN EN 2006
}

\author{
Mercedes Caffaro Rovira (1), Jorge García Pérez (2), Carmen Varela Santos(3), Dionisio Herrera \\ Guibert (3) y Salvador de Mateo Ontañón (3) \\ (1) Programa de Epidemiología Aplicada de Campo (PEAC), Centro Nacional de Epidemiología, Instituto de Salud \\ Carlos III. \\ (2) Subdirección General de Servicios Aplicados Formación e Investigación. Área de Investigación en Telemedicina \\ y Sociedad de la Información. Instituto de Salud Carlos III. \\ (3) Centro Nacional de Epidemiología, Instituto de Salud Carlos III.
}

\section{RESUMEN}

Fundamento: Teniendo en cuenta que una de las misiones de la Red Nacional de Vigilancia Epidemiológica es aportar información para la acción, se hace imprescindible su difusión en tiempo oportuno. El objetivo de este trabajo es conocer el contenido de las páginas web del Centro Nacional de Epidemiología y de los Servicios de Vigilancia Epidemiológica de las Ciudades y Comunidades Autónomas y evaluar su oportunidad en el tiempo.

Métodos: Se realizó una revisión del contenido de las páginas web de los Servicios de Vigilancia durante el período del 3 de abril al 5 de mayo de 2006. La difusión de la información se consideró «en tiempo oportuno» si la consulta durante la semana 17 ofrecía el boletín de la semana epidemiológica 15.

Resultados: El 90\% de los Servicios de Vigilancia epidemiológica tiene página web. El $50 \%$ ofrece el listado de las enfermedades de declaración obligatoria y la definición de caso. Protocolos de actuación el $44 \%$ y tablas dinámicas el 11\%. En el $83 \%$ de las páginas se tiene acceso a los boletines epidemiológicos, el $6 \%$ está actualizado en tiempo oportuno. El $77 \%$ de las páginas ofrece la información semanal de la gripe y de ellas el 78\% en la semana 15. El 72\% tiene un sistema de búsqueda, así como enlaces con otros Servicios de Vigilancia.

Conclusiones: La información difundida por los Servicios de Vigilancia Epidemiológica a través de las páginas web es variable. Es oportuna la difusión de la información de la gripe, no así para el Boletín Epidemiológico.

Palabras clave: Vigilancia epidemiológica. Internet. Difusión de la información.

Correspondencia:

Mercedes Caffaro Rovira.

Programa de Epidemiología Aplicada de Campo

Centro Nacional de Epidemiología

Instituto de Salud Carlos III

Ministerio de Sanidad y Consumo.

Calle Sinesio Delgado, 6

28029 Madrid

Correo electrónico: mcaffaro@isciii.es

\section{ABSTRACT \\ The Spanish Epidemological Surveillance Services Webpages as an Information Dissemination Tool in 2006}

Background: Taking into account that one of the Spanish National Epidemological Surveillance Network's missions is that of providing information for taking action, it is then absolutely essential that its information be disseminated in a timely manner. This study is aimed at ascertaining what information is being published on the National Epidemology Center webpages and on those of the Municipal and Autonomous Community Epidemiological Surveillance Services and assessing the timeliness of the information published.

Methods: A review was conducted of the contents of the Surveillance Services webpages of April 3 - May 5, 2006. The information was considered to be "timely" if the information read on the webpage during Week 17 provided the epidemological week 15 bulletin.

Results: A total of ninety percent $(90 \%)$ of the Services have a webpage, fifty percent $(50 \%)$ of which provide the listing of the compulsorily reportable disease and the case definition, a total of $44 \%$ having protocols for taking action and $11 \%$ having dynamic tables. The epidemological bulletins can be accessed via eight-three percent $(83 \%)$ of the pages, six percent of which are updated in a timely manner. A total of seventy-seven $(77 \%)$ of the pages provide weekly influenza information, seventy-eight percent (78\%) in week 15. A total seventy-two percent $(72 \%)$ have a search system as well as links to other Surveillance Services.

Conclusions: The information provided by the Epidemological Surveillance Services has been found to vary to a certain degree. Influenza information is being disseminated in a timely manner, this however not being the case for the Epidemiological Bulletin.

Key words: Epidemiologic surveillance. Internet. Information dissemination. 


\section{INTRODUCCIÓN}

En 1995 se creó la Red Nacional de Vigilancia Epidemiológica estableciéndose que el Sistema de Vigilancia Epidemiológica debía enmarcarse bajo una estructura descentralizada ${ }^{1}$. A partir de entonces cada una de las 17 Comunidades Autónomas y las 2 Ciudades Autónomas disponen de un Servicio de Vigilancia Epidemiológica, o equivalente, que depende de su Consejería de Sanidad. Por otro lado el Centro Nacional de Epidemiología del Instituto de Salud Carlos III, organismo autónomo del Ministerio de Sanidad y Consumo, tiene la responsabilidad de coordinar la vigilancia epidemiológica que se lleva a cabo en el territorio español.

La Red Nacional de Vigilancia Epidemiológica tiene la misión de aportar información para la acción, de modo que sea útil para la toma de decisiones y los programas de control y de prevención. Para ello es imprescindible que la difusión de la información se establezca en tiempo oportuno entre los diferentes niveles que integran la Red.
La Red esta constituida por varios niveles. El primero es el encargado de realizar la notificación y registro de las enfermedades de declaración obligatoria, función que llevan a cabo los profesionales sanitarios. Los datos registrados se transfieren desde este nivel al siguiente y así sucesivamente (figura 1) Cada uno de los responsables de su respectivo nivel realiza las siguientes funciones : análisis de los datos, toma de decisiones y puesta en marcha de programas de control y prevención. Este proceso requiere una devolución de la información a cada uno de los niveles que integran la Red, lo que se conoce como retroalimentación.

Diferentes autores señalan que la información debe estar disponible para las fuentes que notifican, las personas e instituciones con funciones de planificación, los investigadores y el público en general ${ }^{2}$. En la actualidad esta es la línea de trabajo y parte de la información generada por los Servicios de Epidemiología no se dirige tan solo a los profesionales sanitarios si no que también es accesible a la población general.

Figura 1

Difusión de la información entre los niveles de la Red de Vigilancia Epidemiológica y sus responsables

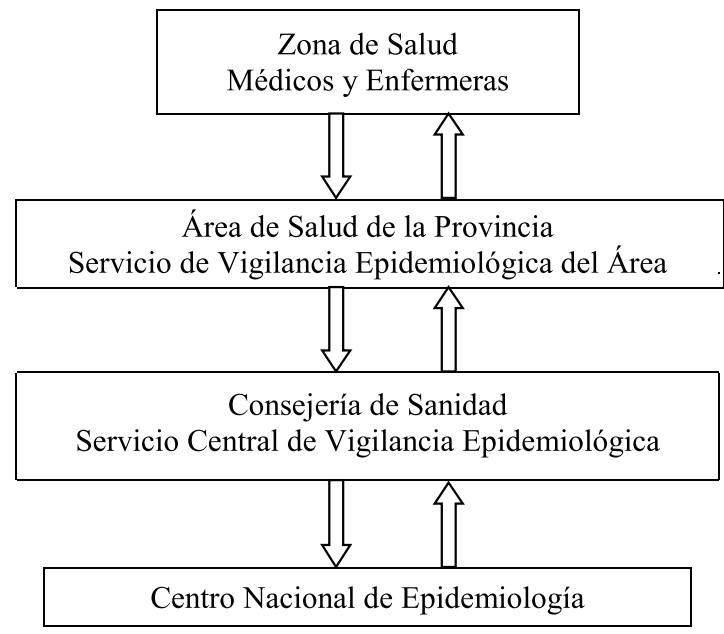


Teniendo en cuenta lo anterior, para que la información generada desde los Servicios de Vigilancia Epidemiológica se difunda a las diferentes audiencias precisa de dos características esenciales:

- De los medios adecuados

- De una divulgación en tiempo oportuno ${ }^{3}$.

El principal documento de comunicación entre la Vigilancia Epidemiológica y los profesionales sanitarios es el Boletín Epidemiológico. Hasta hace unos años su difusión se realizaba en forma impresa. En la actualidad los nuevos medios de comunicación y difusión de la información, internet entre ellos, se han implantado como un recurso más en los Servicios de Vigilancia Epidemiológica, facilitando a los profesionales sanitarios, y usuarios en general, el acceso a la información que generan ${ }^{4}$.

El objetivo de este trabajo es conocer el contenido y la estructura de las páginas web del Centro Nacional de Epidemiología (CNE) y de los Servicios Centrales de Vigilancia Epidemiológica de las Comunidades y Ciudades Autónomas (CCAA), valorar la difusión en tiempo oportuno de los boletines epidemiológicos y del informe semanal de la gripe.

\section{MATERIAL Y MÉTODOS}

Se realizó una revisión, como usuario de internet, de la información difundida en las páginas web de los Servicios de Vigilancia Epidemiológica que integran la Red Nacional, así como de la estructura de dichas páginas web. Se consideraron los siguientes indicadores de buen funcionamiento:

\section{De la información difundida por los Servicios de Vigilancia Epidemiológica}

1. Indicadores de contenido
- Enfermedades de declaración obligatoria (EDO): La existencia de una entrada en la página web de los Servicios de Vigilancia Epidemiológica para las enfermedades de declaración obligatoria: Aparece un listado de enfermedades EDO en la entrada en la página de EDO; se da una definición de caso de cada una de las EDO; se accede a los protocolos establecidos para las EDO; se da información mediante tablas dinámicas en la página web de las EDOS.

- Boletín epidemiológico: Se difunde el Boletín epidemiológico a través de la página web; su periodicidad o frecuencia de la difusión; difusión en tiempo oportuno.

- Sistemas centinelas: si se ofrece información de los sistemas centinelas; si presentan el listado de las enfermedades que se estudian bajo vigilancia centinela; si hay difusión de la información semanal de la gripe y si se difunde en tiempo oportuno.

- SARS y otras alertas: Si las páginas web aportan información sobre el SARS, la gripe aviar, y protocolos de brotes de toxiinfección alimentaría.

- Legislación: si desde la página web de vigilancia tenemos acceso a la legislación que rige la vigilancia epidemiológica de las CCAA y del CNE.

- Ultimas noticias: si en la página web hay una entrada a «ultimas noticias».

2. Otros indicadores

- Vías de comunicación con los Servicios de VE: Si aparece información para establecer comunicación con el Servicio de Vigilancia.

- Usuarios: Usuarios a los cuales va dirigida la información: profesionales, usuarios generales. 
- Actualización: Si la página se encuentra actualizada e indica su fecha de actualización.

- Enlaces: Si desde la página web de vigilancia se pueden establecer enlaces con otras páginas web de Servicios de vigilancia epidemiológica.

\section{Estructura de la página web de los Servicios de Vigilancia Epidemiológica}

La estructura se refiere a la forma que tiene la página web en términos generales, su funcionalidad y sus sistemas de navegación. Interesa conocer la presencia de las herramientas indicadas en la tabla 1.

Para los boletines epidemiológicos y el informe semanal de la gripe se consideró que la información se difundía en tiempo oportuno si la consulta de la página web durante la semana 17 de 2006 (del 23 al 29 de abril) ofrecía el boletín de la semana epidemiológica 15 ( del 9 al 15 de abril), las cuales abarcaban el periodo de estudio.

Los datos obtenidos se analizaron con el programa EXCEL MS Office.

\section{RESULTADOS}

Los Servicios Centrales de Vigilancia Epidemiológica son 20: CNE, 17 CCAA, y 2 Ciudades Autonómicas. El 90\% (18) de ellos tienen una página web (Anexo 1).

Respecto a la información difundida por los Servicios de Vigilancia Epidemiológica: En $9(50 \%)$ páginas web de los Servicios de Vigilancia Epidemiológica se facilita el listado de las enfermedades de declaración obligatoria. Se accede a la definición de caso de las EDO en 9 (50\%) páginas web, a los protocolos de actuación en $8(44 \%)$ y presentan tablas dinámicas 2 CCAA (11\%).

De las páginas web que ofrecen información de las EDOS $8(88 \%)$ presentan conjuntamente el listado de EDO, la definición de caso y los protocolos. Solo 1 CCAA ofrece toda la información analizada.

Las 2 CCAA que presentan tablas dinámicas en vigilancia epidemiológica proporcionan la siguiente información: Una de las CCAA presenta una entrada a un mapa GIS, donde se pueden obtener las tasas de incidencia anuales medias de las EDOS desde 1990 a 2002 por las tres siguientes áreas geo-

Tabla 1

Estructura de la página web: conceptos, definiciones e indicadores

\begin{tabular}{|l|l|l|}
\hline \multicolumn{1}{|c|}{ Concepto } & \multicolumn{1}{|c|}{ Definición } & \multicolumn{1}{c|}{ Indicador } \\
\hline Accesibilidad & $\begin{array}{l}\text { Supone que personas con deficiencias o sin ellas, puedan } \\
\text { acceder a una página y que esta le resulte clara, } \\
\text { comprensible y navegable. }\end{array}$ & $\begin{array}{l}\text { Si la página web tiene algún sello de } \\
\text { accesibilidad. }\end{array}$ \\
\hline Buscador & $\begin{array}{l}\text { Sitio web que está diseñado para que podamos encontrar } \\
\text { los recursos y la información por temas o por palabras } \\
\text { clave. }\end{array}$ & $\begin{array}{l}\text { Se ha cuantificado si en las páginas web } \\
\text { hay un sistema de búsqueda. }\end{array}$ \\
\hline Migas & $\begin{array}{l}\text { Elementos en los sitios web que indican al usuario en } \\
\text { que lugar se encuentra y ofrece poder volver a cualquier } \\
\text { punto anterior con facilidad. }\end{array}$ & $\begin{array}{l}\text { Se ha cuantificado la presencia o no de } \\
\text { migas en las páginas web. }\end{array}$ \\
\hline Mapa Web & $\begin{array}{l}\text { Representación jerárquica de las secciones y contenidos } \\
\text { de un sitio web. }\end{array}$ & $\begin{array}{l}\text { Se ha constatado la presencia o no de } \\
\text { mapas web. }\end{array}$ \\
\hline Web master & $\begin{array}{l}\text { Permite contactar con los técnicos de la página web } \\
\text { mediante su e-mail. }\end{array}$ & $\begin{array}{l}\text { Si las páginas web estudiadas poseen un } \\
\text { web master. }\end{array}$ \\
\hline
\end{tabular}


gráficas; provincias, municipios y área de salud. La otra CCAA da el número de casos desde 1995 a 2005 anuales, semanales o cuatrisemanales por las siguientes áreas geográficas; provincias y municipios.

En cuanto a la difusión de los boletines epidemiológicos 15 páginas web $(83 \%)$ dan acceso a sus respectivos boletines epidemiológicos (figura 2) siendo la periodicidad de su emisión diversa (figura 3). El 6\% de los boletines epidemiológicos se encuentran actualizados en tiempo oportuno.

En 14 páginas web (77\%) se da información sobre los sistemas centinela, pudiendo acceder al informe semanal de la gripe, y de ellos el 78\% se difunde en tiempo oportuno. El 73\% de las páginas que presentan el boletín epidemiológico proporcionan a su vez el informe semanal de la gripe.

Quedan reflejados en la tabla 2 los porcentajes de las otras variables analizadas: alertas, legislación de VE que rige en su ámbito de actuación, últimas noticias, vías de comunicación y actualización.

El $44 \%$ tiene una entrada especifica para profesionales sanitarios aunque puede acceder todo usuario de internet, lo que hace que el $100 \%$ de la información sea accesible a la

Figura 2

Difusión en las páginas web de los Servicios de Vigilancia Epidemiológica de los boletines epidemiológicos $y$ del informe semanal de la gripe

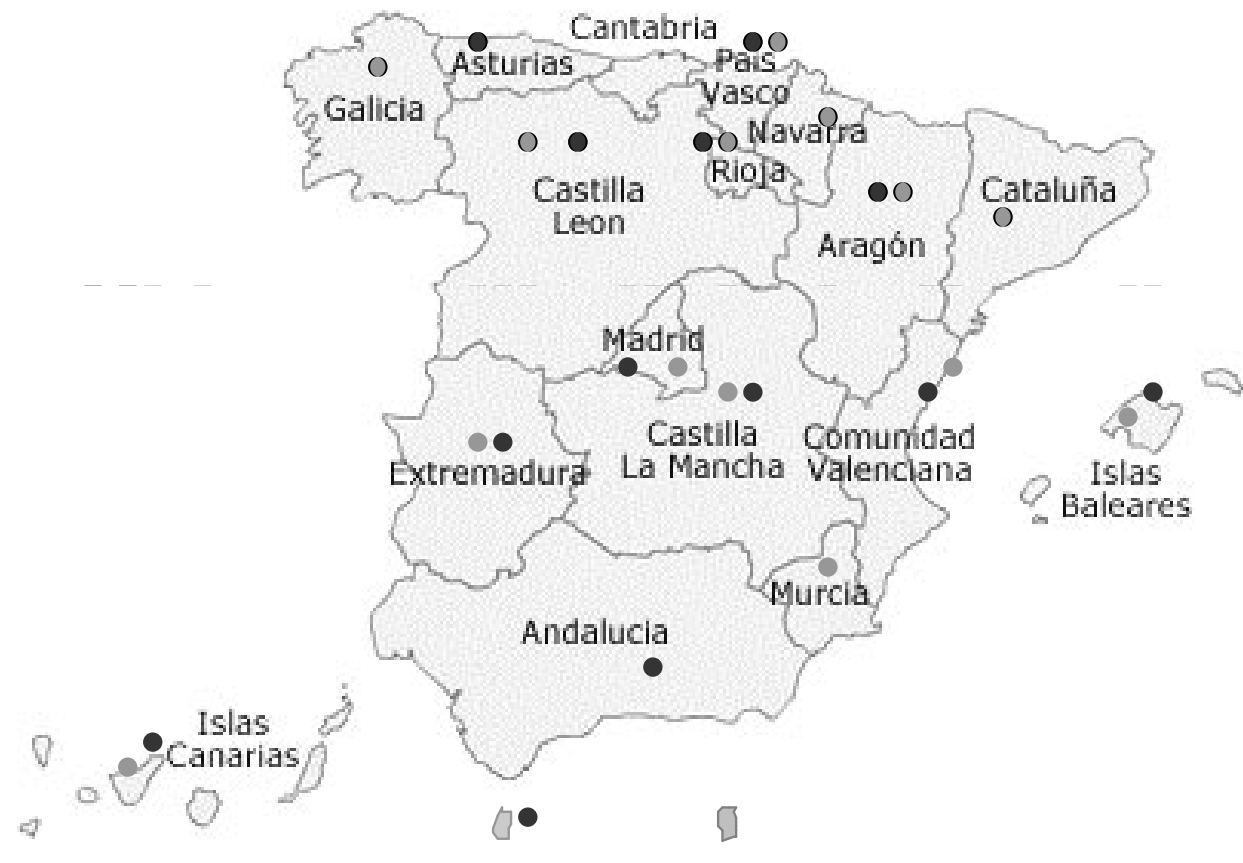

CNE:

O Boletín epidemiológico

- Informe semanal de la gripe 
Tabla 2

Información difundida en las páginas web de los servicios de vigilancia epidemiológica

\begin{tabular}{|l|c|}
\hline \multicolumn{1}{|c|}{ Información } & $\%$ \\
\hline Actualización & $100 \%$ \\
\hline Alertas & $83 \%$ \\
\hline Vías de comunicación & $83 \%$ \\
\hline Ultimas noticias & $72 \%$ \\
\hline Legislación & $38 \%$ \\
\hline
\end{tabular}

población general. En el 94\% de las páginas web, se puede establecer al menos un enlace con otros Servicios de Vigilancia Epidemiológica.

Respecto a la estructura de la página web de los Servicios de Vigilancia Epidemiológica; el $83 \%$ tiene un sello de accesibilidad, el $72 \%$ un sistema de búsqueda automática; migas, el 55\% y mapa web, el 88\%. El 100\% de las páginas cuenta con un web master.

\section{DISCUSIÓN}

Como resultado de nuestro análisis debemos señalar que este estudio es el primero que se realiza en España para conocer la información que difunden los Servicios de Vigilancia Epidemiológica en sus páginas web.

Se puede valorar como muy positivo que el $90 \%$ de los Servicios de Vigilancia Epidemiológica tengan una página web como recurso para difundir su información, lo cual refleja una muy buena incorporación y adaptación a los nuevos medios de comunicación.

Si analizamos la difusión por internet de los boletines epidemiológicos, en España es del $75 \%$, pues se difunden 15 boletines epidemiológicos, de un total de 20 Servicios
Centrales de Vigilancia Epidemiológica. En otros estudios realizados ${ }^{4}$, se obtuvieron porcentajes menores, por ejemplo de los 45 países europeos analizados tan solo $18(40 \%)$ difunden sus boletines epidemiológicos en internet, frente a los países de América que alcanzan el $83 \%$.

La diferente temporalidad en la emisión de los boletines epidemiológicos, y los criterios de nuestra búsqueda puede ser la causante de que su difusión en tiempo oportuno tan solo alcance el $6 \%$.

No obstante para el informe semanal de la gripe, en la semana 15 , se obtuvo una oportunidad del $78 \%$, que podemos considerar bueno.

En cuanto a la variabilidad con la que nos hemos encontrado en el estudio podría ser debida a diferentes razones como: a una dinámica de actualización y rediseño de las páginas, a los diferentes objetivos marcados por los Servicios de Vigilancia Epidemiológica en la difusión de su información, a la diferencia de recursos existentes en los mismos, etc... El análisis de estos factores como posibles causas de la variabilidad pudieran ser la base para un estudio futuro.

Consideramos que una de las ventajas que aportan las páginas web de los Servicios de 
Figura 3

Periodicidad de los Boletines Epidemiológicos

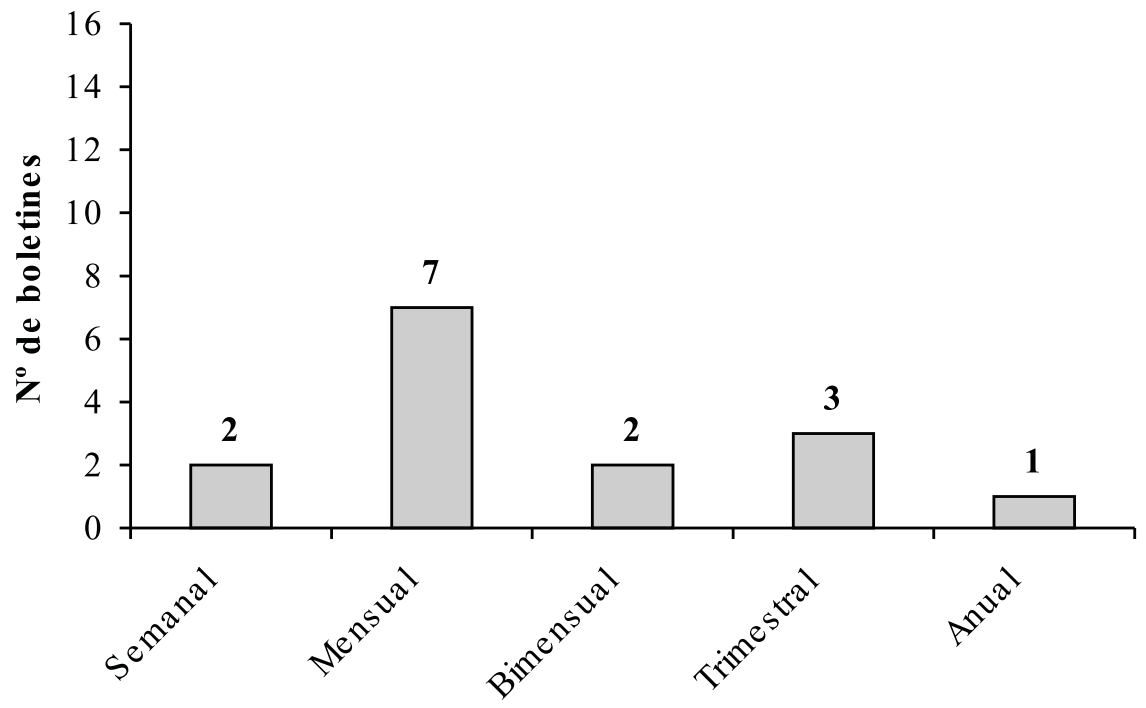

Vigilancia Epidemiológica es la de ofrecer más información que la impresa en los boletines epidemiológicos como protocolos de actuación ante alertas, legislación sobre la vigilancia epidemiológica, ....Así mismo existen otras ventajas como tener acceso al e-mail, teléfono o fax de los Servicios de Vigilancia Epidemiológica, facilitando establecer el contacto con ellos.

Nuestro trabajo ha permitido observar que la estructura de la página web (buscador, migas, mapa web y web master), existen buenos resultados según los estandares internacionales, llegando a porcentajes que van del $55 \%$ en las migas, al $100 \%$ en el web master.

En el apartado de «ultimas noticias» se ha obtenido un resultado (72\%) que podemos considerar satisfactorio, dado la variabilidad de este indicador.

Pensamos que hubiera sido interesante conocer el número de consultas que se hacen a las páginas web y quien las realiza, personal sanitario o población general. Un estudio realizado en Andalucía sobre el informe semanal electrónico de vigilancia epidemiológica, pasó durante su primer mes de funcionamiento, en 1996, de una media de 10 consultas al día, a 101 consultas diarias en enero de $1998^{5}$. Las consultas realizadas a la página web del CNE, desde 1998 a 2004, se han multiplicado por 10 , pasando de 100.000 a cerca de un millón y medio, siendo la página más visitada la que contiene el Boletín Epidemiologico ${ }^{6}$. Estos datos nos indican que con el transcurso de los años se incrementan las consultas por Internet a los Servicios de Vigilancia.

El estudio se ha realizado mediante la entrada a las páginas web de los Servicios de Vigilancia Epidemiológica, quizás la realización de una encuesta dirigida a los diferentes Servicios hubiera proporcionado mejores resultados. No obstante el interés del estudio fue observar lo que localizaría el usuario general de Internet. 
Como resultado del estudio hemos observado limitaciones con las que se pueden encontrar los usuarios para las consultas sobre vigilancia epidemiológica via internet como: la necesidad de tener un ordenador y una conexión a Internet estable, la dificultad, en algunos casos, para localizar las páginas web; los posibles cambios y pérdidas del contenido de la página, con el paso del tiempo; así como el tiempo que se dedica a la identificación inicial en internet en contraste con la recibida en formato papel in situ.

No obstante, pensamos que a pesar de estas limitaciones, este nuevo recurso que se ha incorporado a la Vigilancia Epidemiológica proporciona una mayor difusión de información entre los diferentes niveles de la Red así como a la población general.

Podemos concluir que Internet ha mejorado la difusión, la calidad de la información y la facilidad de acceso a la misma. El estudio ha permitido identificar una variabilidad en cuanto a la información difundida por los diferentes Servicios de Vigilancia. Seria conveniente una mayor homogeneidad en los contenidos de las diversas páginas web que facilitaría el intercambio de información.

Por otro lado se aprecia diferencia de temporalidad en la difusión de los boletines epidemiológicos. Es oportuna la difusión de la información semanal de la gripe. En sentido general la estructura de las páginas web es adecuada. Pensamos que es necesario mantener la divulgación en internet de la información de Vigilancia Epidemiológica y favorecer acciones que faciliten una homogeneidad en determinados aspectos en la información que se difunde.

\begin{tabular}{|c|c|}
\hline CCAA - CNE & Direcciones de las páginas web \\
\hline $\mathrm{CNE}$ & http://www.isciii.es \\
\hline Andalucía & http://www.juntadeandalucia.es/salud \\
\hline Aragón & $\begin{array}{l}\text { http://portal.aragob.es/servlet/page?_pageid }=4329 \& \_ \text {dad=portal30\&_schema=PORTAL30\&_type }=\text { site\&_fsiteid } \\
=707 \& \_f i d=1566512 \& \text { fnavbarid }\end{array}$ \\
\hline Baleares & http://dgsalut.caib.es \\
\hline Canarias & http://www.gobiernodecanarias.org/sanidad \\
\hline Castilla la Mancha & http://www.jccm.es/sanidad \\
\hline Castilla León & http://www.jcyl.es/jcyl-client/jcyl/cs/dgspc?locale=es_ES\&textOnly=false \\
\hline Cataluña & http://www.gencat.net/salut \\
\hline Ceuta & http://www.ciceuta.es \\
\hline C. Valenciana & http://www.san.gva.es/ \\
\hline Extremadura & http://www.juntaex.es/consejerias/syc \\
\hline Galicia & http://www.sergas.es/ \\
\hline Madrid & http://www.madrid.org/sanidad/ \\
\hline Murcia & http://www.murciasalud.es \\
\hline La Rioja & http://www.larioja.org/web/centrales/salud/salud.htm \\
\hline Navarra & http://www.cfnavarra.es/salud \\
\hline País Vasco & http://www.osanet.euskadi.net/r85-3553/es/ \\
\hline P. Asturias & http://www.princast.es/servlet/page?_pageid=2245\&_dad=portal301\&_schema=PORTAL30 \\
\hline
\end{tabular}




\section{AGRADECIMIENTOS}

Al personal de los Servicios Centrales de Vigilancia Epidemiológica. A los compañeros y coordinadores del PEAC.

\section{BIBLIOGRAFÍA}

1. Real Decreto 2210/1995 por el que se crea la Red Nacional de Vigilancia Epidemiológica. BOE 21, 24/1/1996.

2. Ortiz Z, Esandi ME, Bortman M. Módulos de Epidemiología básica y Vigilancia Epidemiológica del Curso de Capacitación en Epidemiología básica y Vigilancia de la salud; 5: Vigilancia de la Salud. $2^{\text {a }}$ Ed; 2004. Disponible en: http://epidemiologia.ar11.toservers. com/pdf/modulos/Modulo\%205-2004.pdf

3. Diagnostico sobre el uso de boletines para la difusión de información epidemiológica en la Región de las Américas. Boletín epidemiológico Organización Panamericana de la Salud, 200324 (2). Disponible en: http:// www.paho.org/Spanish/DD/AIS/be_v24n2cover.htm

4. Woodall J, Aldis R Gaps in global surveillance 1. BioWeapons prevention project. 2003. Disponible en: http://www.bwpp.org/publications/occasional/op001-global-surveillance.pdf

5. Conejo G, Garcia J, Fernández JC, Carmona JC, Delgado E, Guillén J et al. Comunicación; Internet y Vigilancia epidemiológica en Andalucía. Primeras Jornadas Nacionales de Internet y salud. Madrid 1998. http://www. seis.es/inforsaludnet $98 /$ comunicaciones/ 025/Default.htm

6. López-Abente G, Pérez-Gómez B, Rodríguez S, Aragonés N, Póllan M, TelloAnchuela O. El servidor web del Centro Nacional de epidemiología. Evaluación de siete años de funcionamiento. Bol Epidemiol Semanal 2004; 281-288.

7. Zurriaga Lloréns O. Mesa redonda: Internet y la salud de los ciudadanos. La importancia de Internet en la vigilancia en salud pública
Disponible en: http://www.seis.es/inforsaludnet $98 /$ mesas $/ 015 / \mathrm{m} 1505 / \mathrm{m} 1505 . h t m$

8. Merlo Vega JA. La evaluación de la calidad de la información web: aportaciones teóricas y experiencias prácticas. Publicado en Recursos informativos: creación, descripción y evaluación. Mérida: Junta de Extremadura, 2003; 101-110.

9. Comisión de las Comunidades Europeas: Europa 2002: criterios de calidad para los sitios web relacionados con la salud. Bruselas $2002 \mathrm{http}: / /$ www.guiafc.com/documentos/2002-COM-667.pdf

10. Fernández S, Zorrilla B, Ramírez R, Álvarez MC et al. Boletín epidemiológico de la comunidad de Madrid: encuesta sobre su difusión y percepción entre los médicos de atención primaria en el año 2000. Rev Esp Salud Pública 2002; 76: 347-357.

11. Arias B, Fernández de la Hoz K, Domínguez F, Noguerales de la Obra R Et al. Evaluación de un sistema de información en Salud Pública: experiencia en el área 2 de la Comunidad de Madrid. Rev Esp Salud Pública 2002; 76 : 735-742.

12. Fernández F. Las nuevas tecnologías de la información y las comunicaciones en salud. Rev cubana Educ Med Super 2002;16(2): 128-139.

13. Domínguez-Castro A, Ingesta-García A. Evaluación de la calidad de las webs de centros de farmacoeconomía y economía de la salud en internet mediante un cuestionario validado. Gac Sanit 2004;18: 295-304.

14. Mira JJ , Pérez-Jover V, Lorenzo S. Navegando en internet en busca de información sanitaria: no es oro todo lo que reluce. Aten Primaria 2004;33(7):391-9.

15. OMS Surveillance mondiale des maladies infectieuses. Disponible en : http://www. who.int/mediacentre/factsheets/fs200/fr/prin t.html Consultado 24/05/2005.

16. Carnicero J Informes seis. Luces y sombras de la información de salud en Internet $1^{\mathrm{a}}$ ed. Pamplona 2002. 
17. Guía para desarrollo de sitios web. Gobierno de Chile. Capitulo 2: Definición del sitio web. Disponible en: http//www.quiaweb. gob.cl/

18. Gónzalez C, Louro A Serrano J El portal sanitario. Características generales. Cómo desarrollar los Servicios de un portal sanita- rio. Informes SEIS; luces y sombras de la información de salud en Internet. $1^{a}$ ed Pamplona 2002.

19. García M El punto de vista del usuario profesional: Calidad del contenido. Informes SEIS; Luces y sombras de la información de salud en Internet. $1^{\mathrm{a}}$ ed Pamplona 2002. 